\title{
CIRCLE-VALUED MORSE THEORY FOR FRAME SPUN KNOTS AND SURFACE-LINKS
}

\author{
HISAAKI ENDO AND ANDREI PAJITNOV
}

\begin{abstract}
Let $N^{k} \subset S^{k+2}$ be a closed oriented submanifold, denote its complement by $\boldsymbol{C}(\boldsymbol{N})=\boldsymbol{S}^{\boldsymbol{k}+\mathbf{2}} \backslash \boldsymbol{N}$. Denote by $\boldsymbol{\xi} \in \boldsymbol{H}^{\mathbf{1}}(\boldsymbol{C}(\boldsymbol{N}))$ the class dual to $\boldsymbol{N}$. The Morse-Novikov number of $\boldsymbol{C}(\boldsymbol{N})$ is by definition the minimal possible number of critical points of a regular Morse map $C(N) \rightarrow S^{\mathbf{1}}$ belonging to $\xi$. In the first part of this paper we study the case when $N$ is the twist frame spun knot associated to an $\boldsymbol{m}$-knot $\boldsymbol{K}$. We obtain a formula which relates the Morse-Novikov numbers of $\boldsymbol{N}$ and $K$ and generalizes the classical results of D. Roseman and E.C. Zeeman about fibrations of spun knots. In the second part we apply the obtained results to the computation of Morse-Novikov numbers of surface-links in 4-sphere.
\end{abstract}

\section{CONTENTS}

1. Introduction

2. Twist frame spun knots

3. Rotation

4. Surface-links

5. Acknowledgements

References

\section{INTRODUCTION}

1.1. Overview of the article. Let $N^{k} \subset S^{k+2}$ be a closed oriented submanifold, let $C(N)=S^{k+2} \backslash N$ be its complement. The orientation of $N$ determines a cohomology class $\xi \in \boldsymbol{H}^{\mathbf{1}}(\boldsymbol{C}(\boldsymbol{N})) \approx\left[\boldsymbol{C}(\boldsymbol{N}), \boldsymbol{S}^{\mathbf{1}}\right]$. We say that $\boldsymbol{N}$ is fibred if there is a Morse map $f: C(N) \rightarrow S^{1}$ homotopic to $\xi$ which is regular nearby $N$ (see Def 1.1 . and has no critical points. In general a Morse map $C(N) \rightarrow S^{1}$ has some critical points, the minimal number of these critical points will be called the Morse-Novikov number of $\boldsymbol{N}$ and denoted $\mathcal{M N}(\boldsymbol{C}(\boldsymbol{N}))$.

In the first part of this paper we study this invariant in relation with constructions of spinning. The classical Artin's spinning construction [2] associates to each knot $K \subset S^{3}$ a 2 -knot $S(K) \subset S^{4}$. A twisted version of this construction is due to E.C. Zeeman [14]. In [12] D. Roseman introduced a frame spinning construction, and G. Friedman [4] gave a twisted version of generalized Roseman's construction to include twisting.

The input data for twist frame spinning construction is:

(TFS1) A closed manifold $M^{k} \subset S^{m+k}$ with trivial (and framed ) normal bundle.

2010 Mathematics Subject Classification. 57Q45, 57R35, 57R70, 57R45.

Key words and phrases. surface-link, Morse-Novikov number, twist framed spun knots. 
(TFS2) An $\boldsymbol{m}$-knot $\boldsymbol{K}^{\boldsymbol{m}} \subset \boldsymbol{S}^{\boldsymbol{m + 2}}$.

(TFS3) A smooth map $\lambda: M \rightarrow S^{1}$.

To these data one associates an $n$-knot $\sigma(M, K, \lambda$ ), where $n=k+m$ (see Section 2). We prove in Section 2 the following formula:

$$
\mathcal{M N}(C(\sigma(M, K, \lambda))) \leqslant \mathcal{M N}(C(K)) \cdot \mathcal{M N}(M,[\lambda])
$$

(where $\mathcal{M N}(M,[\lambda])$ is the minimal number of critical points of a map $\boldsymbol{M} \rightarrow \boldsymbol{S}^{\mathbf{1}}$ homotopic to $\lambda$.) If $\lambda$ is null-homotopic, we have

$$
\mathcal{M N}(C(\sigma(M, K))) \leqslant \mathcal{M N}(C(K)) \cdot \mathcal{M}(\boldsymbol{M})
$$

(where $\mathcal{M}(\boldsymbol{M})$ is the Morse number of $\boldsymbol{M}$ ). In particular, if $\boldsymbol{K}$ is fibred, then the framed spun knot $\sigma(M, K)$ is fibred (A theorem due to D. Roseman [12]). If in formula (1) the map $l: M \rightarrow S^{\mathbf{1}}$ has no critical points, then the knot $\sigma(M, K, \lambda)$ is fibred, and we recover the classical result of E.C. Zeeman [14]: for any knot its twist-spun knot is fibered. In section 3 we discuss a geometric construction which is related to spinning, namely rotation of a knot $K^{m} \subset S^{m+2}$ around equatorial sphere $\boldsymbol{\Sigma}$ of $S^{\boldsymbol{m + 2}}$. The resulting submanifold $R(K)$ is diffeomorphic to $\boldsymbol{S}^{\mathbf{1}} \times \boldsymbol{S}^{\boldsymbol{m}}$ and is sometimes called spun torus of $\boldsymbol{K}$. We prove that

$$
\mathcal{M N}(\boldsymbol{R}(K)) \leqslant \mathbf{2} \mathcal{M N}(K)+\mathbf{2} .
$$

Section 4 is about Morse-Novikov theory for surface-links. In Subsection 4.1 we introduce a related invariant of surface-links, namely the saddle number $\boldsymbol{s} d(\boldsymbol{F})$ (Definition 4.1) and prove the formula

$$
\mathcal{M N}(\boldsymbol{C}(\boldsymbol{F})) \leqslant \mathbf{2 s d}(\boldsymbol{F})+\chi(\boldsymbol{F})-\mathbf{2} .
$$

In Subsection 4.2 we discuss the case of spun knots. In subsection 4.3 we apply the results of Sections 2 and 3 and the formula (2) to determine the Morse-Novikov numbers of certain surface-links. In [13] K. Yoshikawa introduced a numerical invariant $\operatorname{ch}(\boldsymbol{F})$ of surface-links $\boldsymbol{F}$ and developed a method that allowed him to enumerate all the (weakly prime) surface-links $\boldsymbol{F}$ with $\boldsymbol{c h}(\boldsymbol{F}) \leqslant 10$. In Subsection 4.3 we compute the Morse-Novikov numbers of the majority of the oriented surface-links of the Yoshikawa's table.

1.2. Basic definitions and lower bounds for Morse-Novikov numbers. We start with the definition of a regular Morse map.

Definition 1.1. Let $N^{k} \subset S^{k+2}$ be a closed oriented submanifold. Denote by $\xi \in \boldsymbol{H}^{1}(\boldsymbol{C}(\boldsymbol{N})) \approx\left[\boldsymbol{C}(\boldsymbol{N}), \boldsymbol{S}^{\mathbf{1}}\right]$ the cohomology class dual to the orientation of $\boldsymbol{N}$. A Morse map $f: C(N) \rightarrow S^{1}$ is said to be regular if there is an orientation preserving $C^{\infty}$ trivialisation

$$
\Phi: T(N) \rightarrow N \times B^{2}(0, \epsilon)
$$

of a tubular neighbourhood $\boldsymbol{T}(\boldsymbol{N})$ of $N$ such that the restriction $f \mid(\boldsymbol{T}(\boldsymbol{N}) \backslash N)$ satisfies $f \circ \Phi^{-1}(x, z)=z /|z|$.

An $f$-gradient $v$ of a regular Morse map $f: C(N) \rightarrow S^{1}$ will be called regular if there is a $C^{\infty}$ trivialisation (3) such that $\Phi^{*}(v)$ equals $\left(\mathbf{0}, v_{0}\right)$ where $v_{0}$ is the Riemannian gradient of the function $z \mapsto z /|z|$.

If $f$ is a Morse map of a manifold to $R$ or to $S^{\mathbf{1}}$, then we denote by $\boldsymbol{m}_{p}(f)$ the number of critical points of $f$ of index $p$. The number of all critical points of $f$ is denoted by $\boldsymbol{m}(\boldsymbol{f})$. 
Definition 1.2. The minimal number $m(f)$ where $f: C(N) \rightarrow S^{\mathbf{1}}$ is a regular Morse map is called the Morse-Novikov number of $N$ and denoted by $\mathcal{M N}(C(N))$.

To obtain lower bounds for numbers $\boldsymbol{m}_{p}(f)$ one uses the Novikov homology. Let $\boldsymbol{L}=\mathbb{Z}\left[\boldsymbol{t}, \boldsymbol{t}^{-\mathbf{1}}\right]$; denote by $\widehat{\boldsymbol{L}}=\mathbb{Z}((\boldsymbol{t}))$ and $\hat{\boldsymbol{L}}_{\mathbb{Q}}=\mathbb{Q}((\boldsymbol{t}))$ the rings of all series in one variable $t$ with integer (respectively rational) coefficients and finite negative part. Recall that $\hat{\boldsymbol{L}}$ is a PID, and $\widehat{\boldsymbol{L}}_{\mathbb{Q}}$ is a field. Consider the infinite cyclic covering $\overline{C(N)} \rightarrow C(N)$; the Novikov homology of $C(N)$ is defined as follows:

$$
\widehat{\boldsymbol{H}}_{*}(\boldsymbol{C}(\boldsymbol{N}))=\boldsymbol{H}_{*}(\overline{\boldsymbol{C}(\boldsymbol{N})}) \underset{\boldsymbol{L}}{\otimes} \widehat{\boldsymbol{L}} .
$$

The rank and torsion number of the $\hat{\boldsymbol{L}}$-module $\widehat{\boldsymbol{H}}_{*}(\boldsymbol{C}(\boldsymbol{N}))$ will be denoted by $\hat{\boldsymbol{b}}_{\boldsymbol{k}}(\boldsymbol{C}(\boldsymbol{N}))$, respectively $\hat{\boldsymbol{q}}_{\boldsymbol{k}}(\boldsymbol{C}(\boldsymbol{N}))$. For any regular Morse function $\boldsymbol{f}$ there is a Novikov complex $\mathcal{N}_{*}(f, v)$ over $\widehat{\boldsymbol{L}}$ generated in degree $k$ by critical points of $f$ of index $\boldsymbol{k}$ and such that $\boldsymbol{H}_{*}\left(\mathcal{N}_{*}(f, v)\right) \approx \widehat{\boldsymbol{H}}_{*}(\boldsymbol{C}(\boldsymbol{N}))$. (see [1]). Therefore we have the Novikov inequalities

$$
\sum_{\boldsymbol{k}}\left(\widehat{\boldsymbol{b}}_{\boldsymbol{k}}(\boldsymbol{C}(\boldsymbol{N}))+\hat{\boldsymbol{q}}_{\boldsymbol{k}}(\boldsymbol{C}(\boldsymbol{N}))+\widehat{\boldsymbol{q}}_{\boldsymbol{k}-\mathbf{1}}(\boldsymbol{C}(\boldsymbol{N}))\right) \leqslant \mathcal{M N}(\boldsymbol{C}(\boldsymbol{N})) .
$$

These inequalities, which are far from being exact in general, are however very useful in particular in the case of surface-links (see Section 4 .

\section{TWIST FRAME SPUN KNOTS}

We start with a recollection of the twist frame spinning construction following [12], [5], [4]. See the input data (TFS1) - (TFS3) for this construction on the page 1 . Let $a \in K$. Removing a small open disk $D(a)$ from $S^{m+2}$ we obtain an embedded (knotted) disk $K_{0}$ in the disk $D^{m+2} \approx S^{m+2} \backslash D(a)$. We identify $D^{m+2}$ with the standard Euclidean disk of radius 1 and center $\mathbf{0}$ in $\mathbb{R}^{\boldsymbol{m + 2}}$. We have the usual diffeomorphism

$$
\chi: S^{m+1} \times\left[0,1\left[\stackrel{\approx}{\longrightarrow} D^{m+2} \backslash\{0\}, \quad(x, t) \mapsto t x .\right.\right.
$$

We can assume that $\boldsymbol{K}_{\mathbf{0}} \cap \partial \boldsymbol{D}^{\boldsymbol{m}+\mathbf{2}}$ is the standard sphere $\boldsymbol{S}^{\boldsymbol{m}-\mathbf{1}}$ in $\partial \boldsymbol{D}^{\boldsymbol{m}+\mathbf{2}}=\boldsymbol{S}^{\boldsymbol{m}+\mathbf{1}}$. Moreover, we can assume that the intersection of $\boldsymbol{K}_{\mathbf{0}}$ with a neighbourhood of $\partial \boldsymbol{D}^{m+2}$ is also standard, that is,

$$
K_{0} \cap \chi\left(S^{m+1} \times[1-\epsilon, 1]\right)=\chi\left(S^{m-1} \times[1-\epsilon, 1]\right) .
$$

We have a framing of $M$ in $S^{n}$; combining this with the standard framing of $S^{n}$ in $\boldsymbol{S}^{\boldsymbol{n}+2}$ we obtain a diffeomorphism

$$
\Phi: N\left(M, S^{n+2}\right) \stackrel{\approx}{\longrightarrow} M \times D^{m} \times D^{2}
$$

where $N\left(M, S^{n+2}\right)$ is a regular neighbourhood of $M$ in $S^{n+2}$. We can assume that the restriction of $\Phi$ to $N\left(M, S^{n}\right)$ gives a diffeomorphism

$$
\Phi: N\left(M, S^{n}\right) \stackrel{\approx}{\longrightarrow} M \times D^{m} \times\{0\}
$$

induced by the given framing of $M$. The Euclidean disc $D^{m+2}$ is a subset of $D^{m} \times D^{2}$, so that $K_{0} \subset D^{m} \times D^{2}$.

For $\theta \in S^{1}$ denote by $R_{\theta}$ the rotation of $D^{2}$ around its center. The disc $D^{m+2} \subset$ $D^{m} \times D^{2}$ is invariant with respect to this rotation as well as the intersection 
of $K_{0}$ with a small neighbourhood of $\partial D^{m+2}$. We have $\Phi\left(S^{n} \cap N\left(M, S^{n+2}\right)\right)=$ $M \times D^{m} \times\{\mathbf{0}\}$. Let

$$
\boldsymbol{Z}=\left\{(\boldsymbol{x}, \boldsymbol{y}, \boldsymbol{z}) \mid(\boldsymbol{y}, \boldsymbol{z}) \in \boldsymbol{R}_{\boldsymbol{\lambda}(\boldsymbol{x})}\left(\boldsymbol{K}_{\mathbf{0}}\right)\right\} .
$$

This is an $m$-dimensional submanifold of $M \times D^{m} \times D^{2}$. We define $\sigma(M, K, \lambda)$ as follows

$$
\sigma(M, K, \lambda)=\left(S^{n+2} \backslash N\left(M, S^{n+2}\right)\right) \cup \Phi^{-1}(Z) .
$$

This is the image of an embedded $n$-sphere, knotted in general.

\section{Examples and particular cases.}

1) Let $\operatorname{dim} M=0$, so that $M$ is a finite set; denote by $p$ its cardinality. Then the $n$-knot $\sigma(M, K, \lambda)$ is equivalent to the connected sum of $p$ copies of $K$.

2) If $M$ is the equatorial circle of the sphere $S^{2}$, which is in turn considered as an equatorial sphere of $S^{4}$, and $\lambda(x)=1$, we obtain the classical Artin's construction. If $\lambda: S^{1} \rightarrow S^{1}$ is a map of degree $d$, we obtain the Zeeman's twist-spinning construction [14.

3) If $\boldsymbol{\lambda}(\boldsymbol{x})=\mathbf{1}$ for all $\boldsymbol{x} \in M$ we obtain the Roseman's construction of spinning around the manifold $M[12$. In this case we will denote $\sigma(M, K, \lambda)$ by $\sigma(M, K)$.

Theorem 2.1.

$$
\mathcal{M N}(\sigma(M, K, \lambda)) \leqslant \mathcal{M N}(K) \cdot \mathcal{M N}(M,[\lambda])
$$

(where $[\lambda] \in \boldsymbol{H}^{\mathbf{1}}(\boldsymbol{M}, \mathbb{Z}) \approx\left[\boldsymbol{M}, \boldsymbol{S}^{\mathbf{1}}\right]$ is the homotopy class of $\lambda$ ).

Proof. We will be using the terminology from the above construction of $\sigma(M, K, \lambda)$. We have the standard fibration

$$
\psi_{0}: S^{n+2} \backslash S^{n} \rightarrow S^{1}
$$

obtained from the canonical framing of $S^{n}$ in $S^{n+2}$. Observe that the map $\alpha=$ $\psi_{0} \circ \boldsymbol{\Phi}^{-1}$ is defined by the following formula

$$
\alpha(x, y, z)=\frac{z}{|z|} .
$$

Let $f: S^{m+2} \backslash K \rightarrow S^{1}$ be a Morse map. The restriction of $f$ to the subset $D^{m+2} \backslash K_{0}$ will be denoted by the same letter $f$. We can assume that the function $f$ equals $\alpha$ in a neighbourhood of $\partial D^{m+2}=S^{m+1}$. In particular in a neighbourhood of $\partial D^{m+2}$ we have

$$
f\left(R_{\theta}(p)\right)=f(p)+\theta, \text { for } p \in S^{m+1} \backslash K_{0} .
$$

Define a function $g$ on $M \times D^{m+2} \backslash Z$ by the following formula:

$$
\boldsymbol{g}(\boldsymbol{x}, \boldsymbol{\xi})=\boldsymbol{f}\left(\boldsymbol{R}_{-\boldsymbol{\lambda}(\boldsymbol{x})}(\boldsymbol{\xi})\right)+\boldsymbol{\lambda}(\boldsymbol{x}),
$$

(where $x \in M, \xi \in D^{m+2}$ ). Define a function $\psi$ on the complement $S^{n+2} \backslash \sigma$ by the the following formula:

1) If $p \notin N\left(M, S^{n+2}\right)$, then $\psi(p)=\psi_{0}(p)$.

2) If $\boldsymbol{p} \in \boldsymbol{N}\left(\boldsymbol{M}, \boldsymbol{S}^{\boldsymbol{n + 2}}\right)$, then $\boldsymbol{\psi}(\boldsymbol{p})=\boldsymbol{g}\left(\Phi^{-1}(\boldsymbol{p})\right)$.

We will now prove that if $\boldsymbol{\lambda}$ is a Morse map (this can be achieved by a small perturbation of $\lambda$ ), then $\psi$ is also a Morse map, and the number $\boldsymbol{m}(\boldsymbol{\psi})$ of its critical points satisfy

$$
m(\psi)=m(\lambda) \cdot m(f) .
$$


All the critical points of $\psi$ are in $N\left(M, S^{n+2}\right)$. In this domain the function $\psi$ is diffeomorphic to $g$, and the count of critical points of $g$ is easily achieved with the help of the next lemma.

Lemma 2.2. Let $g_{1}: N_{1} \rightarrow S^{1}, g_{2}: N_{2} \rightarrow S^{1}$ be Morse functions on manifolds $N_{1}, N_{2}$. Let $\boldsymbol{F}: N_{1} \times N_{2} \rightarrow N_{2}$ be a map, such that for each $a \in N_{2}$ the map $x \mapsto F(a, x)$ is a diffeomorphism $N_{2} \rightarrow N_{2}$. Define a function $g: N_{1} \times N_{2} \rightarrow S^{1}$ by the following formula:

$$
g\left(x_{1}, x_{2}\right)=g_{1}\left(x_{1}\right)+g_{2}\left(F\left(x_{1}, x_{2}\right)\right) .
$$

Then $g$ is a Morse function, $\operatorname{Crit}(g)=\operatorname{Crit}\left(g_{1}\right) \times \operatorname{Crit}\left(g_{2}\right)$ and for every $\boldsymbol{a}_{1} \in$ $\operatorname{Crit}\left(g_{1}\right), a_{2} \in \operatorname{Crit}\left(g_{2}\right)$ we have ind $\left(a_{1}, a_{2}\right)=\operatorname{ind}\left(a_{1}\right)+\operatorname{ind}\left(a_{2}\right)$.

Proof. Define a function $g_{0}$ on $N_{1} \times N_{2}$ by the following formula

$$
g_{0}\left(x_{1}, x_{2}\right)=g_{1}\left(x_{1}\right)+g_{2}\left(x_{2}\right) \text {. }
$$

The conclusions of our Lemma hold obviously if we replace $\boldsymbol{g}$ by $\boldsymbol{g}_{\mathbf{0}}$ in the statement of the Lemma. Observe now that the function $g$ is diffeomorphic to $g_{0}$ via the diffeomorphism

The lemma follows.

$$
\left(x_{1}, x_{2}\right) \mapsto\left(x_{1}, F\left(x_{1}, x_{2}\right)\right) .
$$

Corollary 2.3. Let $\boldsymbol{K} \subset \boldsymbol{S}^{3}$ be a classical knot, denote by $\boldsymbol{S}(\boldsymbol{K})$ the spun knot of $\boldsymbol{K}$. Then

$$
\mathcal{M N}(S(K)) \leqslant \mathbf{2} \mathcal{M N}(K)
$$

Proof. In this case $M=S^{\mathbf{1}}$ and $[\lambda]=0$. We have $\mathcal{M N}\left(S^{\mathbf{1}}, \mathbf{0}\right)=\mathbf{2}$ and the result follows. 2.1

The classical theorems concerning fibrations of spun knots follow from Theorem

Corollary 2.4. (D. Roseman [12) If $\boldsymbol{K}$ is fibred, then $\mathcal{M N}(\sigma(M, K))$ is fibred.

Proof. Since $\mathcal{M N}(\boldsymbol{K})=\mathbf{0}$, Theorem 2.1 implies $\mathcal{M N}(\boldsymbol{\sigma}(\boldsymbol{M}, \boldsymbol{K}))=\mathbf{0}$.

Corollary 2.5. (E.C. Zeeman [14]) The $\boldsymbol{d}$-twist spun knot of any classical knot $\mathbf{K}$ is fibred for $\boldsymbol{d} \geqslant 1$.

Proof. Consider a great circle $\Sigma$ in $S^{2}$. The $d$-twist spun knot of $K$ is by definition the $n+1$-knot $\sigma(\Sigma, K, \lambda)$ in $S^{3}$ where $\Sigma \rightarrow \Sigma$ is a map of degree $d$. The assertion follows, since $\mathcal{M N}\left(\boldsymbol{S}^{1}, \lambda\right)=0$.

Remark 2.6. The Zeeman's theorem above generalizes immediately to the following statement: If $\mathcal{M N}(M, \lambda)=0$, then the knot $\sigma(M, K, \lambda)$ is fibred for any knot $K$.

\section{ROTATION}

Let $\boldsymbol{\Sigma}$ be an equatorial sphere of $S^{n+1}$. We can view the sphere $S^{n+1}$ as the union of two discs $D_{+} \cup D^{-}$intersecting by $\boldsymbol{\Sigma}$. Consider $S^{n+1}$ as the equatorial sphere of $S^{n+2}$. The sphere $S^{n+2}$ can be considered as the result of rotation of the disc $\boldsymbol{D}_{+}$around its boundary $\boldsymbol{\Sigma}$. We have the (linear orthogonal) action of $\boldsymbol{S}^{\mathbf{1}}$ on $S^{n+2}$, such that $\boldsymbol{\Sigma}$ is the fixed point set of the action, and the action is free on the rest of the sphere $S^{n+2}$. Let $K^{n-1}$ be an $(n-1)$-knot in $S^{n+1}$. We can assume 

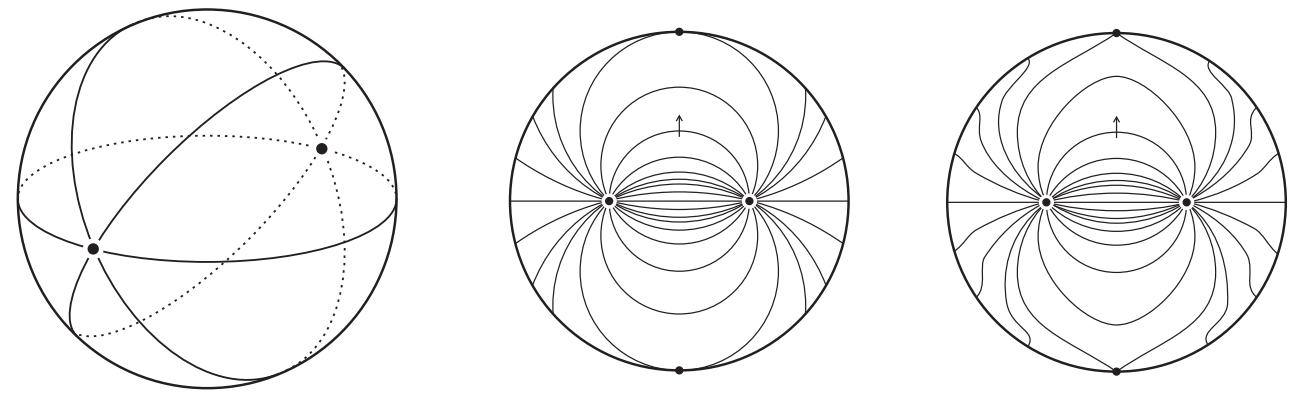

FIGURE 1.

that $K^{n-1} \subset$ Int $D_{+}$. Rotation of $K^{n-1}$ around $\Sigma$ gives a submanifold $R(K)$ of codimension 2 in $S^{n+2}$. The manifold $R(K)$ is diffeomorphic to $S^{1} \times K$. We call this constrution rotation. When $\operatorname{dim} K=1$, the manifold $R(K)$ is sometimes called the spun torus of $\boldsymbol{K}$.

In this section we relate the Morse-Novikov numbers of $\boldsymbol{R}(\boldsymbol{K})$ with those of $\boldsymbol{K}$. The main aim of this section is to prove the following theorem.

\section{Theorem 3.1.}

$$
\mathcal{M N}(R(K)) \leqslant 2 \mathcal{M N}(K)+2 .
$$

To prove the theorem we associate to each given regular Morse function $\phi$ : $S^{n+1} \backslash K^{n-1} \rightarrow S^{1}$ a regular Morse function $R(\phi): S^{n+2} \backslash R\left(K^{n-1}\right) \rightarrow S^{1}$ such that $\boldsymbol{m}(R(\phi))=\mathbf{2} \boldsymbol{m}(\phi)+\mathbf{2}$.

We begin by an outline of this construction for the simplest case when $n=1$ and $K$ consists of two points in $S^{2}$ (Subsection 3.1. In Subsection 3.2 we give a detailed proof of the assertion of the theorem in full generality.

3.1. Rotation of $S^{0}$. Let $K^{0}=\{a, b\} \subset S^{2}$. The manifold $S^{2} \backslash\{a, b\}$ is fibered over $S^{1}$, and the structure of the level lines of this fibration is shown on the figure 1 (left).

Let $D_{-}$be a small 2-disc around any regular point $a$ of $f$. Denote by $D_{+}$the complement $\boldsymbol{S}^{\mathbf{2}} \backslash$ Int $D_{-}$, so that $\boldsymbol{S}^{\mathbf{2}}=\boldsymbol{D}_{+} \cup D_{-}$and the discs $\boldsymbol{D}_{ \pm}$intersect by their common boundary $\Sigma$. Removing $D_{-}$we obtain a map $f: D_{+} \backslash\{a, b\} \rightarrow S^{\mathbf{1}}$; the structure of its level lines is shown on the figure 1 (middle).

The restriction $f \mid \boldsymbol{\Sigma}$ has two non-degenerate critical points: $\boldsymbol{N}$ and $\boldsymbol{S}$. The vector $v$ in the figure depicts the gradient of the map $f$. Applying the rotation construction to $K_{0}$ we obtain a trivial 2-component link $R\left(K^{0}\right)$ in $S^{3}$. Let $F_{0}$ :

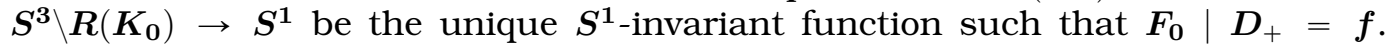
This function is continuous, but not smooth, since its level surfaces have conical singularities in the points of $\Sigma$. To repair this, we will modify the function $f$ in a neighbourhood of $\Sigma$ so that the level lines of the modified function $g: D_{+} \backslash\{a, b\} \rightarrow$ $S^{1}$ are as depicted on the figure 1 (right).

Each non-singular level line intersecting $\Sigma$ is orthogonal to $\Sigma$ at the intersection point. Let $G_{0}: S^{\mathbf{3}} \backslash R\left(K_{0}\right) \rightarrow S^{1}$ be the unique $S^{1}$-invariant function such that $G_{0} \mid D_{+}=g$. Then $G_{0}$ is a $C^{\infty}$ function having two critical points $N$ and $S$. Observe that the descending disc of the critical point $S$ of the function $G_{0} \mid \boldsymbol{\Sigma}$ is 
in $\Sigma$, therefore the descending discs of $G_{0}$ will have the same dimension 1, and ind $_{G_{0}} S=1$. The same reasoning holds for the ascending disc of the critical points $N$, therefore ind $G_{0} N=2$.

3.2. The general case. Let $\Sigma$ be the unit sphere in $\mathbb{R}^{n+2}$, that is,

$$
\Sigma=\left\{\left(x_{0}, \ldots, x_{n+1}\right) \mid x_{0}^{2}+\ldots+x_{n+1}^{2}=1\right\} .
$$

Denote by $\Sigma^{\prime}$ its intersection with the hyperplane $x_{n+1}=0$. Let $a=(\mathbf{0}, \ldots, 0, \mathbf{1})$; for each point $z \in \Sigma^{\prime}$ denote by $C(z)$ the great circle through $a,-a, z$, and by $C^{\prime}(z)$ the closed semicircle containing these three points. The projection $p$ onto the $(n+1)$-th coordinate gives the bijection of $C^{\prime}(z)$ onto the closed interval $[-1,1]$; this bijection is a diffeomorphism when restricted to $C^{\prime}(z) \backslash\{\boldsymbol{a},-\boldsymbol{a}\}$. Let $\boldsymbol{\beta}:[-\mathbf{1}, \mathbf{1}] \rightarrow$ $[-1,1]$ be a diffeomorphism such that $\beta(x)=x$ for $x$ in a neighbourhood of \pm 1 . Then there is a unique diffeomorphism $\bar{\beta}$ of $\Sigma$ onto itself such that for every $z$ the curve $\boldsymbol{C}^{\prime}(\boldsymbol{z})$ is $\overline{\boldsymbol{\beta}}$-invariant and $\boldsymbol{p}(\overline{\boldsymbol{\beta}}(\boldsymbol{v}))=\boldsymbol{\beta}(\boldsymbol{p}(\boldsymbol{v}))$ for every $\boldsymbol{v}$. The diffeomorphism $\overline{\boldsymbol{\beta}}$ will be called the sliding, associated to $\boldsymbol{\beta}$. Observe that every sliding is isotopic to the identity map.

Let $D_{\rho} \subset \Sigma$ be the geodesic disc of radius $\rho$ centered in $-a$. Let

$$
\begin{gathered}
D_{-}=D_{\pi / 2}=\left\{\left(x_{0}, \ldots, x_{n+1}\right) \mid x_{n+1} \leqslant 0\right\}, \\
D_{+}=\left\{\left(x_{0}, \ldots, x_{n+1}\right) \mid x_{n+1} \geqslant 0\right\} .
\end{gathered}
$$

Put $\boldsymbol{\Sigma}_{\rho}=\partial \boldsymbol{D}_{\rho}$. Let $\boldsymbol{N}\left(\boldsymbol{\Sigma}_{\boldsymbol{\rho}}, \boldsymbol{\epsilon}\right)$ denote the geodesic tubular neighbourhood of $\boldsymbol{\Sigma}_{\boldsymbol{\rho}}$. For a given $\rho$ and $\epsilon>0$ sufficiently small there is a sliding $\sigma$ sending $D_{\rho}$ to $D_{-}$ and sending each normal geodesic segment of length $2 \epsilon$ in $N\left(\Sigma_{\rho}, \epsilon\right)$ isometrically to the corresponding normal geodesic segment in $N(\Sigma, \epsilon)$. We have therefore a commutative diagram

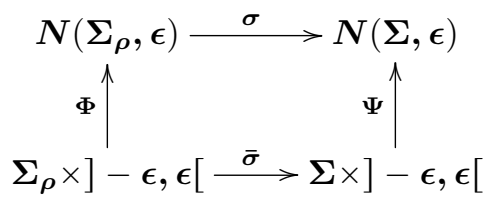

where the vertical arrows are diffeomorphisms and $\overline{\boldsymbol{\sigma}}(\boldsymbol{x}, \boldsymbol{\tau})=(\boldsymbol{\sigma}(\boldsymbol{x}), \boldsymbol{\tau})$.

Let $K$ be an $(n-1)$-knot in $S^{n+1}$ and $\phi: S^{n+1} \backslash K \rightarrow S^{1}$ a Morse map. We can assume that

1) $K \subset$ Int $D_{+}$.

2) $-a \notin$ Critf,

3) the submanifold $\phi^{-1}(\phi(-a))$ is tangent to the hyperplane defined by the equation $\boldsymbol{x}_{\boldsymbol{n}}=\mathbf{0}$.

The restriction $\phi \mid \partial D_{\rho}$ can be considered as a real-valued Morse map. Choosing $\rho$ sufficiently small we can assume that $\phi \mid \Sigma_{\rho}$ is a Morse map having one maximum and one minimum. Denote the function $\phi \circ \Phi$ by $\left.h: \Sigma_{\rho} \times\right]-\epsilon, \epsilon[\rightarrow \mathbb{R}$. For $\rho$ sufficiently small, this function has the following property:

$$
\text { If } \left.\frac{\partial \boldsymbol{h}}{\partial \boldsymbol{t}}(\boldsymbol{x}, \boldsymbol{t})=\mathbf{0}, \text { then } \frac{\partial \boldsymbol{h}}{\partial \boldsymbol{x}}(\boldsymbol{x}, \boldsymbol{t}) \neq \mathbf{0} \text {, where } \boldsymbol{x} \in \boldsymbol{\Sigma}_{\boldsymbol{\rho}}, \boldsymbol{t} \in\right]-\epsilon, \epsilon[.
$$

Consider the restriction of $\phi$ to the subset $S^{n+1} \backslash\left(K \cup D_{\rho}\right)$. Composing $\phi$ with $\sigma^{-1}$ we obtain a function

$$
\phi_{0}: D_{+} \backslash \sigma(K) \rightarrow S^{1} .
$$


This is a Morse map which extends to a geodesic tubular neighbourhood of $\boldsymbol{\Sigma}=$ $\partial \boldsymbol{D}^{+}$, and can be considered as a real-valued Morse function in this neighbourhood. The restriction $\phi_{0} \mid \boldsymbol{\Sigma}$ has two critical points of indices $\boldsymbol{n}$ and $\mathbf{0}$. Denote these critical points by $N$ and $S$, so that ind ${ }_{\phi_{0}} N=n$, ind ${ }_{\phi_{0}} S=0$. The function $h_{0}=\phi_{0} \circ \Psi$ has the following property:

$$
\text { If } \left.\frac{\partial h_{\mathbf{0}}}{\partial t}(x, t)=\mathbf{0} \text {, then } \frac{\partial \boldsymbol{h}_{\mathbf{0}}}{\partial \boldsymbol{x}}(\boldsymbol{x}, t) \neq \mathbf{0} \text {, where } x \in \boldsymbol{\Sigma}, \boldsymbol{t} \in\right]-\epsilon, \epsilon[\text {. }
$$

Now we will modify the function $\phi_{0}$ nearby $\Sigma$. Let $\lambda:[-\epsilon, \epsilon] \rightarrow \mathbb{R}$ be a $C^{\infty}$ function such that $\lambda(t)=|t|$ for $t$ in a neighbourhood of $\{-\epsilon, \epsilon\}$ and $\lambda(t)=t^{2}$ for $|\boldsymbol{t}| \leqslant \boldsymbol{\epsilon} / \mathbf{2}$. Define a function $\boldsymbol{h}_{\mathbf{1}}$ by the following formula:

$$
h_{1}(x, t)=h_{0}(x, \lambda(t)),
$$

and define a function

as follows:

$$
\phi_{1}: D_{+} \backslash \sigma(K) \rightarrow S^{1}
$$

1) if $\boldsymbol{v} \notin N(\boldsymbol{\Sigma}, \epsilon)$, put $\phi_{1}(\boldsymbol{v})=\phi_{0}(\boldsymbol{v})$.

2) if $v \in N(\Sigma, \epsilon), \quad v=\Psi(x, t)$ with $x \in \Sigma, t \in]-\epsilon, \epsilon\left[\right.$, put $\phi_{1}(v)=h_{1}(x, t)$.

Proposition 3.2. The function $\phi_{1}$ has two critical points in $N(\Sigma, \epsilon)$, namely $N$ and $S$. Their indices are equal, respectively, to $n$ and 1.

Proof. The partial derivatives of $\boldsymbol{h}_{1}$ are equal to $\frac{\partial \boldsymbol{h}_{0}}{\partial \boldsymbol{x}}(\boldsymbol{x}, \boldsymbol{\lambda}(\boldsymbol{t}))(\boldsymbol{x}, \boldsymbol{t})$ and $\frac{\partial \boldsymbol{h}_{0}}{\partial \boldsymbol{t}}(\boldsymbol{x}, \boldsymbol{\lambda}(\boldsymbol{t}))$. $\lambda^{\prime}(t)$. For $\boldsymbol{t}=\mathbf{0}$ the second derivative equals 0 , and $\frac{\partial \boldsymbol{h}_{1}}{\partial \boldsymbol{x}}(\boldsymbol{x}, \boldsymbol{\lambda}(\boldsymbol{t}))(\boldsymbol{x}, \mathbf{0})$ vanishes in $\boldsymbol{N}$ and $S$. If $t \neq 0$, then $\lambda^{\prime}(t) \neq 0$, and for $(x, t)$ to be a critical point of $\phi_{1}$ it is necessary that $\frac{\partial \boldsymbol{h}_{0}}{\partial \boldsymbol{t}}(\boldsymbol{x}, \boldsymbol{\lambda}(\boldsymbol{t}))(\boldsymbol{x}, \boldsymbol{t})$ vanish, which implies that $\frac{\partial \boldsymbol{h}_{0}}{\partial \boldsymbol{t}}(\boldsymbol{x}, \boldsymbol{t})(\boldsymbol{x}, \boldsymbol{t}) \neq \mathbf{0}$ (see the property (7)).

Now we are ready to construct a Morse function on the complement to $\boldsymbol{R}(\boldsymbol{K})$. Observe that the knot $K$ is equivalent to the knot $\sigma(\boldsymbol{K})$. By a certain abuse of notation we will replace $\boldsymbol{\sigma}(\boldsymbol{K})$ by $\boldsymbol{K}$, so in particular, $\boldsymbol{K} \subset$ Int $\boldsymbol{D}_{+}$. Add one more coordinate $\boldsymbol{x}_{\boldsymbol{n}+\mathbf{2}}$ and consider the sphere

$$
\Sigma=\left\{\left(x_{0}, \ldots, x_{n+2}\right) \mid x_{0}^{2}+\ldots+x_{n+2}^{2}=1\right\} .
$$

We have $D_{+} \subset S^{n+2}$. The knot $R(K)$ is defined by the following formula:

$$
R(K)=\left\{\left(x_{0}, \ldots, x_{n+2}\right) \mid\left(x_{0}, \ldots, x_{n}, \sqrt{x_{n+1}^{2}+x_{n+2}^{2}}\right) \subset K\right\} .
$$

The circle $S^{1}$ acts on $S^{n+2}$ by rotations in the two last coordinates. Define the Morse function $\phi_{2}$ on the complement to $\boldsymbol{R}(\boldsymbol{K})$ by the two following properties:

1) $\phi_{2} \mid D_{+} \backslash K=\phi_{1}$.

2) $\phi_{2}$ is $S^{1}$-invariant.

The second property implies that

$$
\phi_{2}\left(x_{0}, \ldots, x_{n+2}\right)=\phi_{1}\left(x_{0}, \ldots, x_{n}, \sqrt{x_{n+1}^{2}+x_{n+2}^{2}}\right) .
$$

Observe that the property 2) of the function $\phi_{1}$ guarantees that $\phi_{2}$ is $C^{\infty}$ on the subset $\boldsymbol{S}^{n+2} \backslash R(K)$.

Proposition 3.3. 1) $\operatorname{Crit}\left(\phi_{2}\right)=S^{1} \cdot \operatorname{Crit}\left(\phi_{1}\right) \cup\{N, S\}$, 
2) The critical points $N$ and $S$ are non-degenerate, and

$$
\text { ind } \phi_{\phi_{2}} N=\operatorname{ind}_{\phi_{1}} N=n \text {, ind } \phi_{2} S=\operatorname{ind}_{\phi_{1}} S+1=2 \text {. }
$$

Proof. The point 1) is easy to deduce from the definition of $\phi_{2}$. As for the indices of the critical points observe that the descending disc of the critical point $N$ in $N(\Sigma, \epsilon)$ belongs to the sphere $\boldsymbol{\Sigma}$ which is fixed by the action of $S^{\mathbf{1}}$. Thus the index of $N$ does not change when we replace $\phi_{1}$ by $\phi_{2}$. A similar argument applies to the ascending disc of $\boldsymbol{S}$, and this implies the rest of the proposition.

Each critical point of $\phi_{1}$ gives rise to a circle of critical poins of $\phi_{2}$. Using the same method, as in the previous work of the authors, we wwe perturb the function $\phi_{2}$ in a neighbourhood of each of these critical circles, and obtain finally a regular Morse function $\boldsymbol{R}(\phi)$ on the complement to $\boldsymbol{R}(\boldsymbol{K})$ such that

$$
\# \operatorname{Crit}(\boldsymbol{R}(\phi))=2 \# \operatorname{Crit}\left(\phi_{2}\right)+2 .
$$

This completes the proof of Theorem 3.1 .

3.3. 4-thread spinning. In this subsection we give a brief description of one more construction of surface-links. Let $L \subset S^{\mathbf{3}}$ be a classical link and $\phi: S^{\mathbf{3}} \backslash L \rightarrow S^{\mathbf{1}}$ a Morse map. Let $p, q \in L$ and let $\gamma:[0,1] \rightarrow S^{3}$ be a $C^{\infty}$ curve joining $p$ and $q$ and belonging entirely to one of the regular level surfaces $\phi^{-1}(\lambda)$ of the map $\phi$. We assume morover that $\operatorname{Im} \gamma \cap \boldsymbol{L}=\{\boldsymbol{p}, \boldsymbol{q}\}$, and that $\boldsymbol{\gamma}^{\prime}(\mathbf{0})$ and $\gamma^{\prime}(\mathbf{1})$ are not tangent to $\boldsymbol{L}$. Let $\boldsymbol{D}$ be a small neighbourhood of $\operatorname{Im} \gamma$ diffeomorphic to a 3-disc. Denote by $\boldsymbol{\Sigma}$ its boundary. We can assume that $\boldsymbol{L} \cap \boldsymbol{\Sigma}$ consists of four points and that $\boldsymbol{L}$ is orthogonal to $\Sigma$ at each of these points. Denote by $S_{0}^{2}$ the 2 -sphere with 4 points removed. Recall that there is a standard Morse function $\phi_{0}$ on $S_{0}^{2}$ having 2 critical points of indices 1. We can assume that the restriction of $\phi$ to $\Sigma \backslash L$ is diffeomorphic to $\phi_{0}$.

Remove the interior of $D$ from $S^{3}$ and rotate the remaining manifold $S^{3} \backslash \operatorname{Int} D$ around $\Sigma$. We obtain the sphere $S^{4}$; the subset which is spun by $L \backslash \operatorname{Int} D$ during the rotation is an embedded 2 -surface in $S^{4}$.

We call this construction 4-thread spinning to distinguish it from the usual spinning, and denote the resulting surface-link by $\boldsymbol{S}^{\prime}(\boldsymbol{L})$. If $\boldsymbol{p}$ and $\boldsymbol{q}$ are on different connected components of $\boldsymbol{L}$, then the number of connected components of $\boldsymbol{S}^{\prime}(\boldsymbol{L})$ is the same as for $\boldsymbol{L}$. If $\boldsymbol{p}$ and $\boldsymbol{q}$ are in different connected components of $\boldsymbol{L}$, then the number of connected components of $S^{\prime}(\boldsymbol{L})$ equals that of $\boldsymbol{L}$ increased by 1 . Applying the same method as in the Subsection 3.2 we can construct a Morse function $\widetilde{\phi}$ on $S^{4} \backslash S^{\prime}(L) \rightarrow S^{1}$ such that $m(\widetilde{\phi})=m(\phi)+2$.

\section{Corollary 3.4.}

$$
\mathcal{M N}\left(S^{\prime}(L)\right) \leqslant 2 \mathcal{M N}(L)+2 .
$$

\section{SURFACE-LiNKS}

In this section we develop circle-valued Morse theory for surface-links.

4.1. Motion pictures and saddle numbers. Let $\boldsymbol{F}$ be a surface-link, that is, a closed oriented 2-dimensional $C^{\infty}$ submanifold of $S^{4}$. We can assume $F \subset \mathbb{R}^{4}$.

Choose a projection $p$ of $\mathbb{R}^{4}$ onto a line. Assume that the critical points of the function $p \mid \boldsymbol{F}$ are non-degenerate. Denote by $\operatorname{sdl}(\boldsymbol{F})$ the minimal number of saddle points of $\boldsymbol{p} \mid \boldsymbol{F}$ over all the projections $\boldsymbol{p}$. 
Definition 4.1. A saddle number $s d(F)$ is the minimum of numbers $s d l\left(F^{\prime}\right)$ where $\boldsymbol{F}^{\prime}$ ranges over all surface-links $\boldsymbol{F}^{\prime}$ ambiently isotopic to $\boldsymbol{F}$.

The invariant $\boldsymbol{s d}(\boldsymbol{F})$ is closely related to the $\boldsymbol{c h}$-index of $\boldsymbol{F}$, introduced and studied by K. Yoshikawa in [13. In particular, we have $s \boldsymbol{d}(\boldsymbol{F}) \leqslant \boldsymbol{c h}(\boldsymbol{F})$. In order to relate the number $\boldsymbol{s d}(\boldsymbol{F})$ to $\mathcal{M N}(\boldsymbol{K})$ we will reformulate the definition of the saddle number.

Let $F \subset S^{4}$ be a surface-link. The equatorial 3 -sphere $\Sigma^{3}$ of the standard Euclidean sphere $S^{4}$ divides $S^{4}$ into two parts:

$$
S^{4}=D_{+}^{4} \cup D_{-}^{4}, \text { with } D_{+}^{4} \cap D_{-}^{4}=\Sigma^{3} .
$$

We assume that $\boldsymbol{F}$ is included in $\operatorname{Int}\left(\boldsymbol{D}_{-}^{4}\right)$ and $\boldsymbol{F}$ does not include the centre of $D_{-}^{4}$. Perturbing the embedding $F \subset D_{-}^{4}$ if necessary, we can assume that the restriction $\rho=\left.r\right|_{F}$ of the radius function $r: D_{-}^{4} \rightarrow[0,1]$ is a Morse function. The family $\left\{\left(r^{-1}(t), \rho^{-1}(t)\right)\right\}_{t \in[0,1]}$ of possibly singular links can be drawn as a motion picture (see [8], Chapter 8). Each singularity of a link in the family corresponds to a critical point of $\rho$. A critical point of $\rho$ of index $0(1,2$, respectively) is called minimal point (saddle point, maximal point, respectively) of $\rho$, which is represented by a minimal band (saddle band, maximal band, respectively) in (a modification of) the motion picture.

It is clear that the minimal number of the saddle points for all such Morse functions $\rho$ is equal to $s d(F)$.

Theorem 4.2. $\mathcal{M N}(F) \leqslant 2 \operatorname{sd}(F)+\chi(F)-2$.

Proof. Since $\rho$ is a Morse function, the manifold $D_{-}^{4} \backslash \operatorname{Int} N(F)$ admits a handle decomposition with one $\mathbf{0}$-handle and $\boldsymbol{m}_{\boldsymbol{i}}(\boldsymbol{\rho})(\boldsymbol{i}+\mathbf{1})$-handles for $\boldsymbol{i} \in\{\mathbf{0}, \mathbf{1}, \mathbf{2}\}$ (see [7], and also [6], Proposition 6.2.1).

The exterior $\boldsymbol{E}(\boldsymbol{F})=\boldsymbol{S}^{\mathbf{4}} \backslash \operatorname{Int} \boldsymbol{N}(\boldsymbol{F})$ of $\boldsymbol{F}$ is obtained by attaching a 4-handle $D_{+}^{4}$ to $D_{-}^{4} \backslash \operatorname{Int} \boldsymbol{N}(\boldsymbol{F})$. Since $D_{-}^{\mathbf{4}} \backslash \operatorname{Int} \boldsymbol{N}(\boldsymbol{F})$ is connected, there is a 3-handle in $D_{-}^{4} \backslash \operatorname{Int} \boldsymbol{N}(\boldsymbol{F})$ which connects $\partial \boldsymbol{N}(\boldsymbol{F})$ with $\partial \boldsymbol{D}_{-}^{4}$. Thus the 3 -handle cancels the 4-handle $D_{+}^{4}$ (see [9], Section 5). Turning the handlebody upside down, we obtain a dual decomposition of $\boldsymbol{E}(\boldsymbol{F})$ and a corresponding Morse function $\boldsymbol{f}: \boldsymbol{E}(\boldsymbol{F}) \rightarrow \mathbb{R}$ which is constant on $\partial \boldsymbol{E}(\boldsymbol{F})$ and the following Morse numbers: $\boldsymbol{m}_{\mathbf{1}}(f)=\boldsymbol{m}_{\mathbf{2}}(\rho)-\mathbf{1}$, $m_{2}(f)=m_{1}(\rho), m_{3}(f)=m_{0}(\rho), m_{4}(f)=1$.

Using the argument from work of the second author [10], p. 629, we can deform the real-valued Morse function $f$ to a circle-valued regular function $\phi: E(F) \rightarrow$ $S^{1}$, such that $m_{k}(f)=m_{k}(\phi)$ for every $k$. Consider the function $-\phi$, which has one critical point of index 0 . Applying the cancellation of this local minimum, we obtain a Morse function $\psi: \boldsymbol{E}(\boldsymbol{F}) \rightarrow \boldsymbol{S}^{1}$ belonging to the class $-\boldsymbol{\xi}$, and such that $m_{0}(\psi)=0, m_{1}(\psi)=m_{3}(f)-1, m_{2}(\psi)=m_{2}(f), m_{3}(\psi)=m_{1}(f), m_{4}(\psi)=0$. Put $g=-\psi$. Then we have

$$
\begin{gathered}
m_{0}(g)=m_{4}(g)=0, \quad m_{1}(g)=m_{2}(\rho)-1, \\
m_{2}(g)=m_{1}(\rho), \quad m_{3}(g)=m_{0}(\rho)-1 .
\end{gathered}
$$

Observe that $m_{0}(\rho)-m_{1}(\rho)+m_{2}(\rho)=\chi\left(S^{2}\right)=2$, therefore the total number of critical points of $g$ equals $2 m_{1}(\rho)$. Choosing the function $\rho$ with $m_{1}(\rho)=s d(F)$ we accomplish the proof.

Corollary 4.3. Let $\boldsymbol{K} \subset S^{4}$ be a 2-knot. Then $\mathcal{M N}\left(C_{K}\right) \leqslant 2 s d(K)$. 
Proposition 4.4. Let $\boldsymbol{F} \subset S^{4}$ be the trivial $\boldsymbol{k}$-component surface-link. Then $\mathcal{M N}(F)=4 k-2-\chi(F)$.

Proof. It is not diffcult to show that $\widehat{b}_{\mathbf{1}}(C(F)) \geqslant k-1, \widehat{b}_{\mathbf{3}}(\boldsymbol{C}(\boldsymbol{F})) \geqslant k-1$. Therefore for every regular Morse map $f: C(F) \rightarrow S^{1}$ we have $m_{1}(f)+m_{3}(f) \geqslant$ $2(k-1)$. Assuming $m_{0}(f)=m_{4}(f)=0$ we have $m_{1}(f)-m_{2}(f)+m_{3}(f)=2-\chi(F)$, and $\mathcal{M N}(C(F)) \geqslant 4 \boldsymbol{k}-\mathbf{2}-\chi(\boldsymbol{F})$; this lower bound coincides with the upper bound derived from Theorem 4.2 ,

4.2. Spun knots. Let $K$ be a classical knot in $S^{3}$ denote by $S(K)$ the corresponding spun knot.

Proposition 4.5. If $\boldsymbol{K}$ is a non-fibered knot of tunnel number 1, then $\mathcal{M N}\left(S^{4} \backslash S(K)\right)=$ 4.

Proof. Recall that $\mathcal{M N}\left(S^{4} \backslash S(K)\right) \leqslant \mathbf{2} \mathcal{M N}(\boldsymbol{K})$ (Corollary[2.3. In the paper [10] of the second author it is shown that $\mathcal{M N}(K) \leqslant 2 t(K)$, hence $\mathcal{M N}(S(K)) \leqslant 4$ by Corollary 2.3. Put $\boldsymbol{G}=\boldsymbol{\pi}_{1}\left(\boldsymbol{S}^{\mathbf{3}} \backslash \boldsymbol{K}\right)$, then $\boldsymbol{\pi}_{1}\left(\boldsymbol{S}^{\mathbf{4}} \backslash \boldsymbol{S}(\boldsymbol{K})\right)$; let $\boldsymbol{H}=[\boldsymbol{G}, \boldsymbol{G}]$. Let $f: S^{\mathbf{4}} \backslash S(K) \rightarrow S^{\mathbf{1}}$ be a regular Morse map without minima and maxima. If $m_{1}(f)=\mathbf{0}$, then a standard Morse-theoretic argument applied to the infinite cyclic cover of $S^{\mathbf{4}} \backslash \boldsymbol{S}(\boldsymbol{K})$ implies that $\boldsymbol{H}$ is finitely generated, which is impossible, since $K$ is not fibred. Therefore $m_{1}(f) \geqslant 1$, and similarly, $m_{3}(f) \geqslant 1$, hence $m_{2}(f) \geqslant 2$ and the proposition is proved.

4.3. Surface-links of Yoshikawa's table. Yoshikawa [13] suggested a method for enumerating surface-links. To each surface-link $\boldsymbol{F}$ he associated a natural number $\operatorname{ch}(\boldsymbol{F})$. His methods allowed him to make a list of all (weakly prime) surfacelinks $\boldsymbol{F}$ with $\operatorname{ch}(\boldsymbol{F}) \leqslant 10$. It is clear from the definition of the invariant $\operatorname{ch}(\boldsymbol{F})$ that we have $\boldsymbol{s d}(\boldsymbol{F}) \leqslant \boldsymbol{c h}(\boldsymbol{F})$. In the rest of this section we assume that the reader is familiar with Yoshikawa's work, and with his terminology. There are 6 two-knots in Yoshikawa's table, namely

$$
0_{1}, 8_{1}, 9_{1}, 10_{1}, 10_{2}, 10_{3} .
$$

The trivial 2 -knot $0_{1}$ is obviously fibred. The knots $8_{1}$ and $10_{1}$ are spun knots of the trefoil knot and respectively of the figure $8 \mathrm{knot}$, thus both $\mathbf{8}_{1}$ and $\mathbf{1 0}_{1}$ are fibred by [1].

The case of $9_{1}$ is more complicated. The saddle number of this 2 -knot is 2 . Therefore $\mathcal{M N}\left(\mathbf{9}_{\mathbf{1}}\right) \leqslant 4$. Using the presentation of the fundamental group of the complement to $9_{1}$ (see [13]) and Poincaré duality properties it is easy to compute the Novikov numbers of $\mathbf{9}_{1}$. Namely we have $\widehat{\boldsymbol{q}}_{\mathbf{1}}=\mathbf{1}, \widehat{\boldsymbol{q}}_{\mathbf{2}}=\widehat{\boldsymbol{q}}_{\mathbf{3}}=\mathbf{0}$. Therefore

$$
2 \leqslant \mathcal{M N}\left(9_{1}\right) \leqslant 4 \text {. }
$$

The 2-knot $10_{2}$ is the 2-twist-spun knot of the trefoil knot, hence fibered by Zeeman's theorem [14]. Similarly, $\mathbf{1 0}_{3}$ is fibered, being the 3 -twist spun of the trefoil knot.

The surface-link $6_{1}^{0,1}$ is the result of spinning of the Hopf link which is fibred (see the left of Figure 2) therefore $\mathcal{M N}\left(6_{1}^{0,1}\right)=0$.

The surface-link $\mathbf{8}_{1}^{1,1}$ is the spun torus of the Hopf link. Applying Theorem 3.1 we get the upper bound $\mathcal{M N}\left(8_{1}^{1,1}\right) \leqslant 2$. Computing the Euler charcateristic implis the inverse inequality, so $\mathcal{M N}\left(8_{1}^{1,1}\right)=2$. 

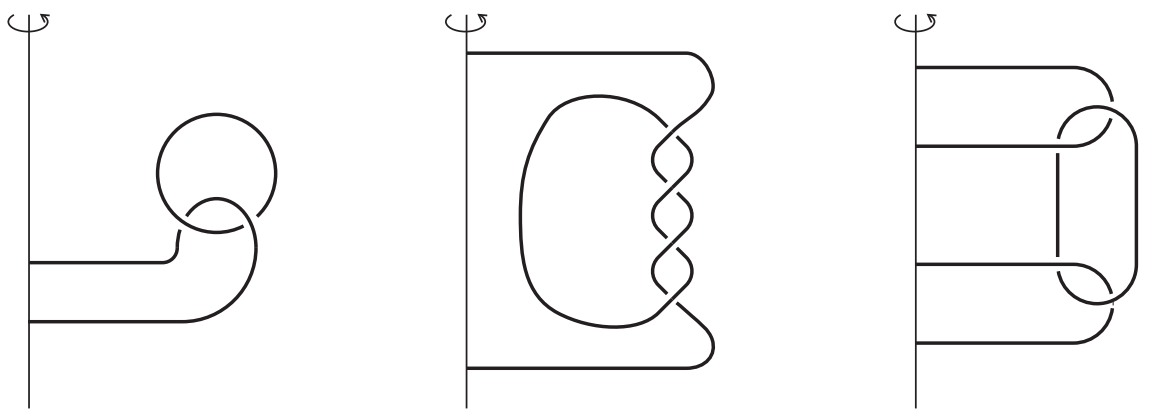

FIGURE 2.

The same argument applies to the surface-link $10_{1}^{1}$, which is the spun torus of the trefoil knot, see the figure 2 (middle), so that $\mathcal{M N}\left(10_{1}^{1}\right)=2$.

The surface-link $10_{1}^{0,1}$ is the result of spinning of the link $4_{1}^{2}$ which is fibred, therefore $\mathcal{M N}\left(\mathbf{1 0}_{\mathbf{1}}^{\mathbf{0}, \mathbf{1}}\right)=\mathbf{0}$.

The case of the surface-link $\boldsymbol{F}=\mathbf{1 0}_{1}^{\mathbf{0 , 0 , 1}}$ is more complicated. This surface-link is the result of 4-threaded spinning of the connected sum $L$ of two copies of the Hopf link, see Figure 2 (right) and applying Corollary 3.4 we deduce $\mathcal{M N}(\boldsymbol{F}) \leqslant 2$. The computation of Euler characteristic gives the lower bound 2 for the MorseNovikov number, thus $\mathcal{M N}\left(\mathbf{1 0}_{1}^{\mathbf{0}, 0,1}\right)=\mathbf{2}$.

\section{ACKNOWLEDGEMENTS}

This work was accomplished when the second author was visiting the Tokyo Institute of Technology in 2016 with the support of the JSPS fellowship. The first author was partially supported by JSPS KAKENHI Grant Numbers 25400082 , 16K05142. The second author thanks the Tokyo Institute of Technology for support and warm hospitality.

\section{REFERENCES}

[1] J.J. Andrews, D. W. Sumners, On higher-dimensional fibered knots, Trans. Amer. Math. Soc., 153 (1971), 415-426.

[2] E. Artin, Zur Isotopie zweidimensionalen Flächen im $\boldsymbol{R}_{4}$, Abh. Math. Sem. Univ. Hamburg 4 (1926), $174-177$.

[3] D.B.A. Epstein, Linking spheres, Proc. Cambridge Phil. Soc. 56 (1960), 215-219.

[4] G. Friedman, Alexander polynomials of non-locally-flat knots, Indiana Univ. Math. J. 52 (2003), 14791578.

[5] G. Friedman, Knot Spinning, Handbook of Knot Theory, Elsevier, 2005, ch.4.

[6] R. E. Gompf and A. I. Stipsicz, 4-manifolds and Kirby calculus, Graduate Studies in Mathematics 20, American Mathematical Society, 1999.

[7] C. McA Gordon, Homology of groups of surfaces in the 4-sphere, Math. Proc. Camb. Phil. Soc, 89, $113-117$.

[8] S. Kamada, Braid and knot theory in dimension four, Math. Surveys Monogr. 95, Amer. Math. Soc., Providence, RI, 2002.

[9] J. Milnor, Lectures on $\boldsymbol{h}$-cobordism theorem, Notes by L. Siebenmann and J. Sondow, Princeton Univ. Press, Princeton, New Jersey, 1965.

[10] A. Pajitnov, On the tunnel number and the Morse-Novikov number of knots, Algebraic \& Geometric Topology 10 (2010) 627635. 
[11] A. Pajitnov, Circle-Valued Morse Theory (de Gruyter Studies in Mathematics 32).

[12] D. Roseman, Spinning knots about submanifolds; spinning knots about projections of knots, Topology and Appl. 31 (1989), 225241.

[13] K. Yoshikawa, An enumeration of surfaces in four-space, Osaka J. Math. 31 (1994), 497-522.

[14] E. C. Zeeman, Twisting spun knots Trans. Amer. Math. Soc. 115 (1965), 471-495.

Department of Mathematics Tokyo institute of Technology 2-12-1 Ookayama, Meguro-ku TOKYO 152-8551 JAPAN

E-mail address: endo@math.titech.ac.jp

Laboratoire Mathématigues Jean Leray UMR 6629, Faculté des Sciences, 2, Rue de la Houssinière, 44072, NANTES, CEDEX

E-mail address: andrei.pajitnov@univ-nantes.fr 